\title{
Kinetic models of peroxidase activity in potato leaves infected with late blight based on hyperspectral data
}

\author{
Qinyu $\mathrm{Li}^{1}$, Yaohua $\mathrm{Hu}^{1,2,3^{*}}$ \\ (1. College of Mechanical and Electronic Engineering, Northwest A\&F University, Yangling 712100, China; \\ 2. Key Laboratory of Agricultural Internet of Things, Ministry of Agriculture, Yangling 712100, China; \\ 3. Shaanxi Key Laboratory of Agricultural Information Perception and Intelligent Service, Yangling 712100, China)
}

\begin{abstract}
Potato late blight, which is caused by Phytophthorainfestans (Mont.) de Bary, is a worldwide devastating disease for potato. It decreased yields of potato and caused unpredictable losses all over the world. Various simple statistical methods and forecasting models have been developed to predict and manage potato late blight. Meanwhile, there is a rising need to develop prediction models reflecting peroxidase (POD) activity, which is an important health index that varies with infection and correlated with stress resistance in plants. Thus, the aim of this research was to develop kinetic models to predict POD activity. Infection-induced changes in potato leaves stored in an artificial climate chest at $25^{\circ} \mathrm{C}$ were analyzed using hyperspectroscopy. Four prediction models were developed by using linear partial least squares (PLS) and nonlinear support vector machine (SVM) methods based on the full spectrum and effective wavelengths. The effective wavelengths were selected by the successive projection algorithm (SPA). In this study, the prediction model developed by means of SPA-SVM method obtained the best performance, with a $R_{p}$ (correlation coefficient of prediction) value of 0.923 and a $R M S E p$ (root mean square error of prediction) value of 24.326. Five-order kinetics models according to the prediction model were developed, and late blight disease can be predicted using this model. This study provided a theoretical basis for the prediction of latencies of late blight.
\end{abstract}

Keywords: POD (peroxidase) activity, kinetic model, potato leaves, late blight, hyperspectral data, latency prediction DOI: $10.25165 /$ j.ijabe. 20191202.4574

Citation: Li Q Y, Hu Y H. Kinetic models of peroxidase activity in potato leaves infected with late blight based on hyperspectral data. Int J Agric \& Biol Eng, 2019; 12(2): 160-165.

\section{Introduction}

Potato (Solanum tuberosum) is one of major foods in the world $^{[1]}$. It is the fourth major staple crop in China and thus important to resolve food-storage issues. According to the latest data from the Ministry of Agriculture of China, the potato planting area will be expanded to one hundred million acres by $2020^{[2,3]}$. Potato late blight, which is caused by phytopathogenic oomycetes, has become the most serious disease of this worldwide food crop, and it has the potential to reduce potato production and lower or even destroy the quality of potatoes. For plants, the development of disease symptoms is correlated with changes in the content of reactive oxygen species (ROS) ${ }^{[4-6]}$. An excessive accumulation of ROS is toxic and always has a negative impact on the plant. Meanwhile, peroxidase (POD) activity plays a central role in counteracting ROS stress, and it can be used to evaluate plant resistance since POD is a chemical defense enzyme that can prevent oxidative damage under different stresses. POD activity information is pivotal for predicting potato leaf disease. Traditional method for POD determination in leaves is chemistry titration, but its time consuming and labor intensive characteristics restricts its application in POD activity detection, as late blight can

Received date: $2018-08-12 \quad$ Accepted date: $2018-12-21$

Biographies: Qinyu Li, Graduate student, research interest: mechatronics, Email: qingyu_li19930203@163.com.

*Corresponding author: Yaohua Hu, Professor, research interest: mechatronics and quick acquisition of agricultural information. College of Mechanical and Electronic Engineering, Northwest A\&F University, Yangling 712100, China. Tel: +86-29-87092391, Email: huyaohua@nwsuaf.edu.cn. develop rapidly and easily cause a pandemic ${ }^{[7,8]}$. Therefore, a rapid method of detecting POD activity in potato leaves is required to achieve real-time monitoring throughout the various stages of the disease. The hyperspectral technique has been widely applied in other agricultural fields ${ }^{[9,10]}$, including determining of the nitrogen nutrition, chlorophyll and water statuses in leaves. A hyperspectral curve-fitting model was established by Champagne for estimating plant water content ${ }^{[11]}$. Based on hyperspectral indices, Sonobe et al. ${ }^{[12]}$ studied leaf chlorophyll concentrations with different leaf types in deciduous forests. Stroppiana et al. ${ }^{[13]}$ built a regression model between the canopy hyperspectral data and nitrogen and chlorophyll contents of paddy rice. However, few studies have focused on detecting POD changes in leaves based on hyperspectral data. The objectives of this study were: (1) to optimize the hyperspectroscopy model of POD activity of potato leaves infected by late blight to predict POD activity changes during infection in an artificial climate chest; (2) to build a kinetic model of POD activity according to the hyperspectroscopy data and infection time under an artificial climate chest temperature; and (3) to predict late blight disease severity according to the POD activity.

\section{Materials and methods}

\subsection{Sample preparation}

Potatoes varieties of XIAZHAI8, QINGTIAN and QILONG were used as samples. The potatoes were planted in a greenhouse in Yangling, Shaanxi Province, China in July 2017. The temperature was maintained at $23^{\circ} \mathrm{C} \pm 2^{\circ} \mathrm{C}$ during the day time, $13^{\circ} \mathrm{C} \pm 2^{\circ} \mathrm{C}$ at night. In the stage of tuber formation and growth, the maximum soil water holding capacity was maintained at $83 \% \pm 3 \%$, and remained at about $70 \% \pm 3 \%$ before harvest. If it 
was rainy or cloudy over two days, 1500 lumens of light were added from 20:00 to 22:00 during the night. Three months later, 120 large and undamaged potato leaves for each variety, total of 360 leaves for all three varieties were picked from the stems. Each leaf was labeled before spectral measurement. Disinfected cotton balls saturated with water were used to cover each leaf petiole.

The samples were randomly divided into six groups, and each group had 20 samples for each potato variety. After acquiring the hyperspectral data and POD activities of the first group, the other five groups were inoculated with late blight fungus at a dose of $100 \mu \mathrm{L}$ per leaf, and measured every $24 \mathrm{~h}$. The late blight pathogen was provided by the College of Plant Protection, Northwest A \& F University. The inoculated positions were located between blade secondary veins. The samples were stored in an artificial climate chamber (PRX-250B, SAIFU in Ningbo) at $25^{\circ} \mathrm{C}$ with $80 \%$ relative humidity. The experiment was finished on the sixth day.

\subsection{Spectral measurements}

The hyperspectral imaging system was used to acquire spectral information of leaves, which is mainly composed of a spectrometer (V10E-QE, ZoLix in Beijing), camera, electric mobile platform, light source and computer. The effective waveband of the system ranged from 375 to $1012 \mathrm{~nm}$, and the spectral resolution was $2.8 \mathrm{~nm}$. All parts except for the computer were placed in a black box to eliminate external light interference during measurement. Certain parameters of the system were set before data acquisition. In the experiment, the parameters were set as follows: $50 \mathrm{~ms}$ exposure time, $20 \mathrm{~mm} / \mathrm{s}$ movement speed of the electric mobile platform and $65 \mathrm{~cm}$ distance from samples to lens. In addition, the system warmed up for $30 \mathrm{~min}$ to prevent drift and reduce experimental error, and the camera was calibrated with a white and dark reference to exclude noise influence. The computer model is Lenovo XiaoXin Air 14. The hardware environment is intel(R) Core (TM) i5-7200U CPU @ 2.5 GHz 8.00GB RAM. The software environment is Windows 10 operating system, and the Python version is 3.6 in Spyder Programming editor. The calibration method followed previous studies $^{[14-16]}$.

On the first day, the spectra of 120 samples were obtained, and the twenty samples in group one was adopted to measure the POD activity. On the second day, the spectra of the remaining 100 samples were obtained, and the twenty samples in group two were adopted to measure the POD activity. On the sixth day, only twenty samples were left for spectra collection and POD activity measurement. Therefore, the samples in group one had one spectrum, and the samples of group six had six spectra. Moreover, to decrease error, three positions on each sample were measured to obtain average spectra.

\subsection{Determination of POD activity in infected potato leaves}

Immediately after the daily spectra were recorded, the POD activity of twenty potato leaves samples was measured using a microplate reader with a 96-cell plate. The specific method was as follows: POD activities were assayed in crude homogenates obtained by grinding $0.2 \mathrm{~g}$ leaf samples with $1.25 \mathrm{~mL}$ extraction buffer $(0.05 \mathrm{~mol} / \mathrm{L}$ phosphate buffer solution, $\mathrm{pH}=7.0)$, and then the homogenates were centrifuged (15000 r/min, $15 \mathrm{~min})$ and the supernatants were collected. All procedures were performed at $4^{\circ} \mathrm{C}$. The reaction mixtures included $1.95 \mathrm{~mL}$ phosphate buffer solution, $1 \mathrm{~mL} \quad 0.3 \% \quad \mathrm{H}_{2} \mathrm{O}_{2}$ solution and $0.05 \mathrm{~mL}$ enzyme homogenates. The optical densities of the solutions were measured at a wavelength of $240 \mathrm{~nm}$. The sample treatment method was previously reported ${ }^{[17]}$. The optical density decreased by 0.01 per minute with one unit of enzyme in each reaction mixture at $\mathrm{pH} 7.0$ and $25^{\circ} \mathrm{C}$. A blank sample was used as reference. The POD activity was determined by the following equation:

$$
P O D=\frac{(A o-A s) \times V t}{F W \times V s \times t}
$$

where, $P O D=$ POD activity, $\mathrm{U} \cdot \mathrm{FW} /(\mathrm{g} \cdot \mathrm{h}) ; A o=$ the absorbance of the contrast in the light condition; $A s=$ absorbance of the sample; $V t=$ bulk volume of the extract, $\mathrm{mL} ; V_{S}=$ crude enzyme cost, $\mathrm{mL}$; $t=$ light application time of the chromogenic reaction, min; and $F W=$ sample weight, g.

\subsection{Spectral data analysis}

Regions of interest (ROIs) were selected in the diseased leaf parts, and average spectral data were extracted with ENVI 4.8. To highlight the spectral information, preprocessing methods were applied to eliminate the spectral baseline shift, noise influence and other measurement errors ${ }^{[18,19]}$. Various spectral preprocessing methods were investigated for building partial least squares (PLS) models and SVM models, including Savitzky-Golay (SG) Smoothing, Median Filter Smoothing, Moving Average Smoothing, Gaussian Filter Smoothing, Normalization, Multiple Scattering Correction (MSC) and Standard Normal Variate (SNV) methods. Pretreatment techniques, such as SG smoothing, MSC, SNV and normalization, were used to remove noise and other factors included in the spectra $^{[20,21]}$. The spectra processed by SG smoothing and MSC methods had similar curve shapes as the raw spectrum, whereas spectra processed by the SNV and normalization methods had different curve shapes from the raw spectrum.

Variable selection methods were also necessary to reduce the dimensionality and complexity of the input variables (hyperspectral data). The successive projection algorithm (SPA) method is a new forward-loop variable selection method that can effectively extract wavelengths from the overlapping spectrum with an emphasis on minimizing collinearity and improving the modeling conditions $^{[22,23]}$. Linear and nonlinear calibration methods were used to build a prediction model of POD information by hyperspectral data. The PLS analysis was generally used for linear calibrations, and the SVM method was investigated for nonlinear calibrations ${ }^{[24]}$. After six days, the 90 undamaged leaves were randomly selected as the calibration set for each variety, and the remaining 30 samples were taken as the prediction set for each variety. The calibration set was used to establish a stable and general model, and the prediction set was used to evaluate the model performance. Different PLS models and SVM models were built using the preprocessing methods mentioned above. The optimal preprocessing method achieved a higher correlation coefficient $(R)$ and lower RMSE. For the PLS models, the input $X$-variables (hyperspectral data) were extracted into new latent variables to present the most relevant information from the original spectra. A full cross-validation process was used to ensure the stability and robustness of the PLS models.

Python 3.6 was used to conduct PLS and SVM, and the sklearn framework was used for fast data analysis. In the PLS model, plsregress function was used, Ncomp is the number of factors or potential variables that set to 8 , Xloadings is ncomp factor load number that set to 10 . In the SVM model, the best production was obtained when regularization parameter $\mathrm{C}$ value is 4.5 and $\mathrm{RBF}$ nuclear function parameter gamma value is 0.8 . Larger $\mathrm{C}$ value 
leads to easier model over fitting. Larger gamma results more support vectors, while small gamma results better model generalization. However, if gamma is too small, the model will actually degenerate into a linear model. The predictive performance quality was evaluated according to correlation coefficient $(R)$ and RMSE. In this study, SPA method was used as the wave number selection method to choose spectra to ensure the accuracy of modeling and quickness of the operation.

\subsection{Development of the model based on chemical kinetics}

Plant health condition related changes are chemical, physical and microbial changes that can be described by kinetic model chemical reactions. Changes in the plant health condition during infection may be related to kinetic characteristics, such as reaction rate constants and activation energies ${ }^{[25,26]}$. Most plant health indexes changes shown in the scatter diagram can be described by the exponential function or power function.

\section{Results and discussion}

\subsection{Pretreatment of spectral data}

The PLS and SVM techniques were used to establish a model to correlate POD activity with the hyperspectral data. The results showed that spectra with the Median Filter pretreatment technique was better than that of the other method, the model based on that technique did not show any under-fitting or over-fitting phenomena and had a small degree of dispersion. The sample values varied from $44.459 \mathrm{mg} / 100 \mathrm{~g}$ to $537.198 \mathrm{mg} / 100 \mathrm{~g}$ during infection; thus, the root mean square error (RMSE) was relatively large. This finding shows that the scattering from the uneven distribution of internal particles and particle size in the potato leaf sample had an effect on the near-infrared spectra. The Median Filter method was selected as the pretreatment technique. As shown in Table 1, the best PLS model performance for the prediction set was preprocessed by the Median Filter Smoothing method $(R p=0.778$, $\left.R M S E_{P}=40.215\right)$, and closely followed by the Moving Average Smoothing method. Median Filter Smoothing and Moving Average Smoothing were selected as the preprocessing methods.

Table 1 PLS model performance of different preprocessing methods

\begin{tabular}{lcccc}
\hline \multicolumn{1}{c}{ Preprocessing methods } & $R_{C}$ & $R M S E_{C}$ & $R_{P}$ & $R M S E_{P}$ \\
\hline Original Spectra & 0.768 & 41.659 & 0.721 & 45.785 \\
Savitzky-Golay Smoothing (SG) & 0.783 & 42.784 & 0.706 & 46.636 \\
Median Filter Smoothing & 0.865 & 40.452 & 0.787 & 40.668 \\
Moving Average Smoothing & 0.825 & 47.266 & 0.725 & 49.314 \\
Gaussian Filter Smoothing & 0.803 & 43.385 & 0.704 & 42.371 \\
Normalization & 0.816 & 45.632 & 0.718 & 48.642 \\
Multiplicative Scatter Correction (MSC) & 0.810 & 42.312 & 0.738 & 42.406 \\
Standard Normal Variate (SNV) & 0.806 & 46.952 & 0.746 & 43.528 \\
\hline
\end{tabular}

According to hyperspectral data preprocessed by seven different methods, the SVM models of POD activity were built based on the full spectra. The result of SVM model was better than that of the PLS model. The $R c, R p$ in Table 2 were generally bigger than that of Table 1, which means the accuracy of the prediction model of SVM was higher than that in PLS. The $R M S E c$, RMSEp of Table 2 were generally lower than that of Table 1 , which means the stability of the prediction model of SVM was higher than that of PLS. Furthermore, Median Filter Smoothing method had the highest $R c, R p$ and the lowest RMSEc, RMSEp, this method was selected as the optimal preprocessing method and used in the subsequent analysis.
Table 2 SVM model performance of different preprocessing methods

\begin{tabular}{lcccc}
\hline \multicolumn{1}{c}{ Preprocessing Methods } & $R_{C}$ & $R M S E_{C}$ & $R_{P}$ & $R M S E_{P}$ \\
\hline Original Spectra & 0.852 & 33.491 & 0.820 & 35.317 \\
Savitzky-Golay Smoothing (SG) & 0.874 & 34.552 & 0.837 & 38.326 \\
Median Filter Smoothing & 0.972 & 27.201 & 0.894 & 28.318 \\
Moving Average Smoothing & 0.917 & 31.712 & 0.853 & 36.203 \\
Gaussian Filter Smoothing & 0.885 & 32.596 & 0.852 & 37.617 \\
Normalization & 0.843 & 39.875 & 0.849 & 33.241 \\
Multiplicative Scatter Correction (MSC) & 0.861 & 35.961 & 0.846 & 38.625 \\
Standard Normal Variate (SNV) & 0.892 & 37.694 & 0.871 & 30.453 \\
\hline
\end{tabular}

\subsection{Selection of effective wavelengths}

The SPA algorithm was used to get the effective wavelengths. The calculation process was achieved by Python software, and the result is shown in Figure 1. The number of optimal characteristic wavelengths is determined by the RMSE of the model which tends to be stable. The number of characteristic wavelengths can be determined by SPA algorithm, the minimum and maximum selected bands in the experiment were 1 and 30, respectively. Therefore, the final number of wavelengths was five. From Figure 1, the bands with characteristic wavelengths are $540 \mathrm{~nm}, 680 \mathrm{~nm}, 863 \mathrm{~nm}$, $897 \mathrm{~nm}$, and $941 \mathrm{~nm}$ respectively. These five bands were sensitive to POD activity changes and included the most effective hyperspectral information. These selected effective wavelengths were used as input variables to establish the PLS and SVM models, and these models were then adopted to determine the POD activity.

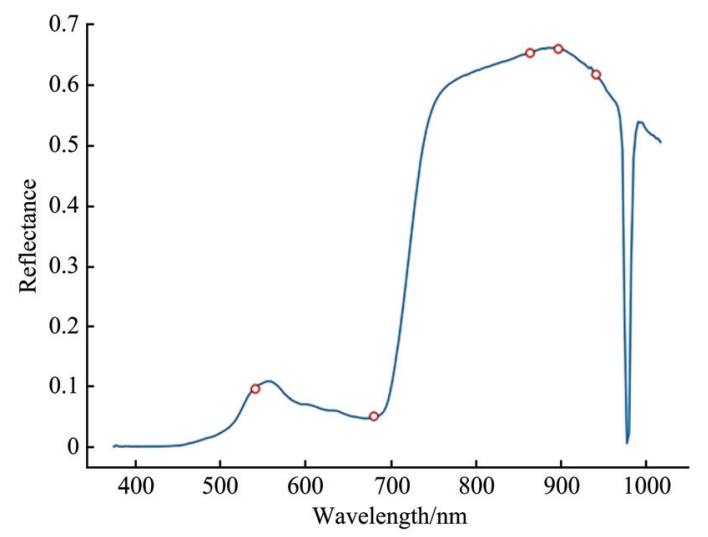

Figure 1 Effective wavelengths selected by the SPA using Python software

The prediction results for POD activity are shown in Table 3. In the SPA-PLS model, different latent variables were extracted and a cross-validation process was developed for a stable performance. The predicted set attained a good performance because of the stability of the model. However, a larger difference in the correlation coefficients was observed between the calibration and prediction sets, which indicated that the PLS model might not be the best model to predict the POD activity in potato leaves with late blight. The wavelength selection method for the SPA-SVM model achieved a better performance than that of the PLS model. The SVM model can exploit latent nonlinear information for the selected effective wavelengths. Thus, the SVM model could be the optimal model for POD activity prediction.

Table 3 Performance of different prediction models by SPA

\begin{tabular}{cccccc}
\hline \multirow{2}{*}{ Models } & \multicolumn{2}{c}{ Calibration set } & & \multicolumn{2}{c}{ Prediction set } \\
\cline { 2 - 5 } \cline { 3 - 5 } & $R_{C}$ & $R M S E_{C}$ & & $R_{P}$ & $R M S E_{P}$ \\
\hline PLS & 0.839 & 32.518 & & 0.817 & 34.195 \\
SVM & 0.941 & 22.613 & & 0.923 & 24.326 \\
\hline
\end{tabular}




\subsection{Change in POD activity of potato leaves after infection}

Figure 2 showed the spectral curve changes for potato leaves after infection. All spectral curves had the same trend. The spectral reflectance peak was at approximately $550 \mathrm{~nm}$. When potato leaves developed from healthy to infectious diseases, the chlorophyll content of leaves gradually decreased. Therefore, the absorption of green light $(\lambda=500-560 \mathrm{~nm})$ decreased $^{[27]}$, while the reflection was strength. Meanwhile the blue color of the leaves increased, the absorption of light $(\lambda=490-500 \mathrm{~nm})$ increased, while the reflection was weaken. In the near-infrared region $(\lambda>780 \mathrm{~nm})$, the spectral reflectance was decreasing as time went on since late blight destroyed potato leaf tissue cells and stopped photosynthesis. The continuous decomposition of synthetic products severely damaged leaves, further decreased the spectral reflectivity of the heavy potato.

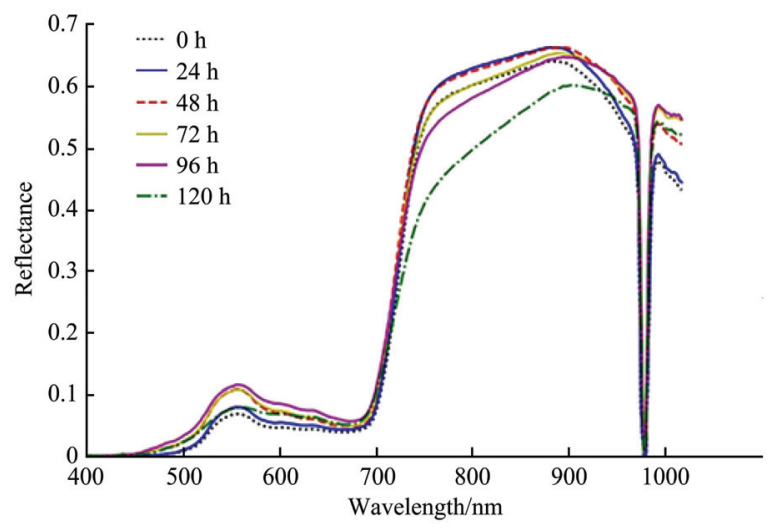

a. Spectral curve of QILONG

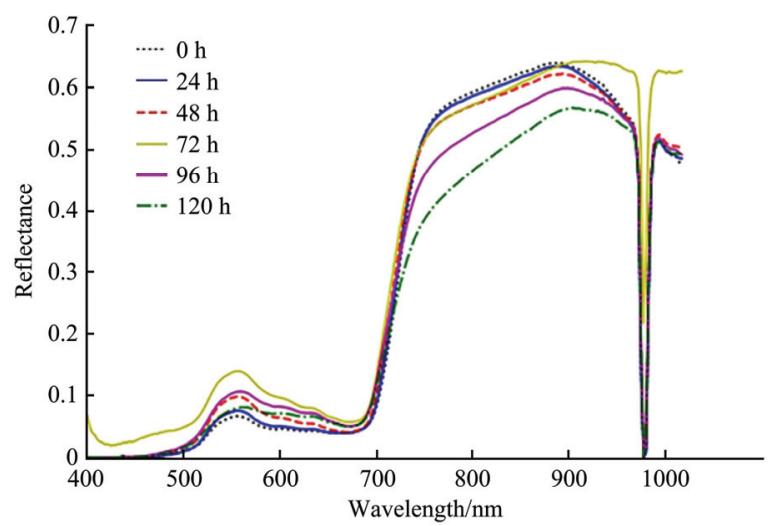

b. Spectral curve of QINGTIAN

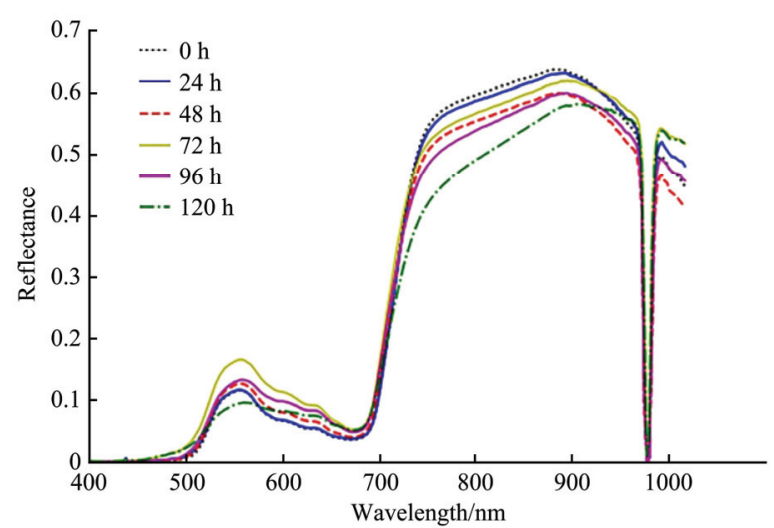

c. Spectral curve of XIAZHAI8

Figure 2 Spectral curve of three variety of leaves' affected region from 0 to $120 \mathrm{~h}$ after infection

The initial POD activity values of the infected potato leaves were calculated using the hyperspectral model and the spectra of each set of infected potato leaves from the first day to the fifth day (Figure 3). The line graphs of the prediction set were shown in Figure 3, which indicates that the POD value initially decreased, then increased, and eventually decreased after reaching a peak value. These changes were inconsistent with the degree of disease. Therefore, distinguishing the disease severity level only according to the POD value was difficult. However, other external symptoms can be used in conjunction with the POD value to diagnose potato late blight. The POD value can be calculated by kinetic prediction models according to the accumulated infection days, and then the degree of disease progression can be easily predicted combined with other external symptoms of potato late blight.
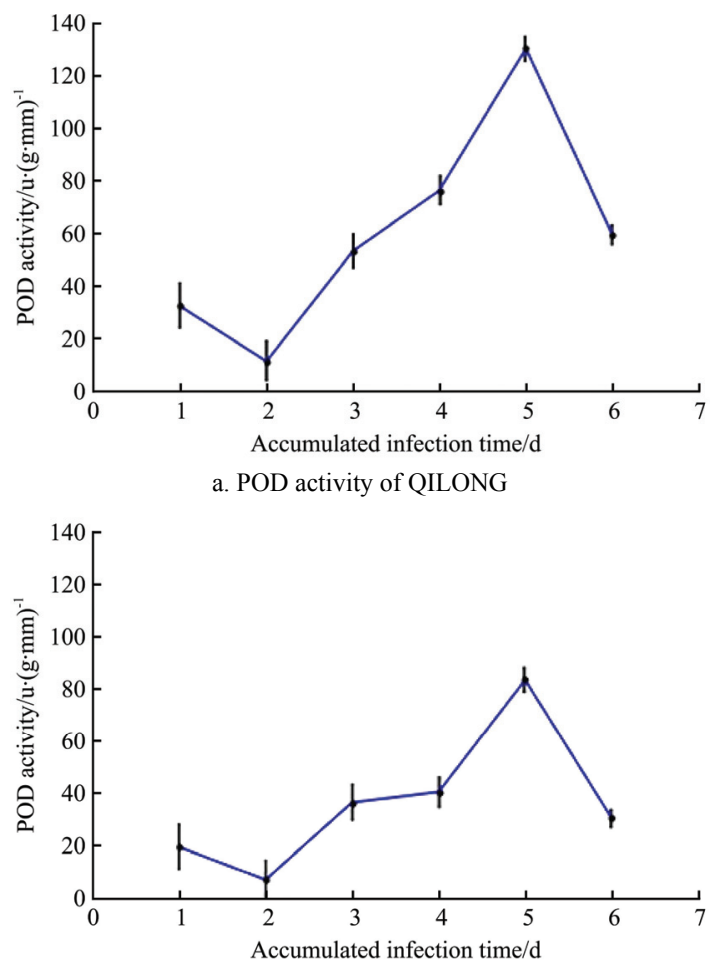

b. POD activity of QINGTIAN

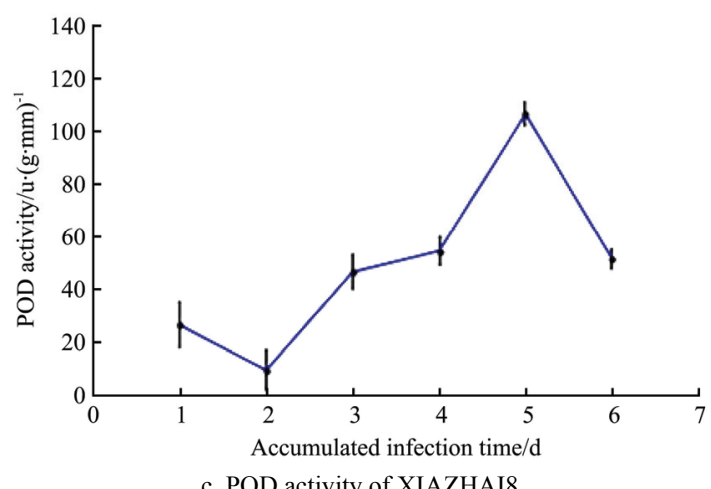

Figure 3 Average change trends of POD activity in the leaves of three potato varieties based on the accumulated infection time

\subsection{Kinetic models for determining POD activity}

POD activity-related changes are chemical, physical and microbial changes that can be described by kinetic model chemical reactions. Changes in POD activity during infection may be related to kinetic characteristics, such as the reaction rate constants and activation energies. Python is a programming language, was used to get the scatter diagram of POD activity. After using os.open fuction to get the data in Excel, Pyplot fuction was then been applied to describe the relationship between POD activity and 
time under the Spyder compiler by GDAL, which can automatically fit the function within the specified error area. The error area was for $10^{-5}$. Figure 4 showed a five-order reaction model for three types of leaves. Thus, the change in POD activity in the infected potato leaves likely followed a five-order kinetics reaction.

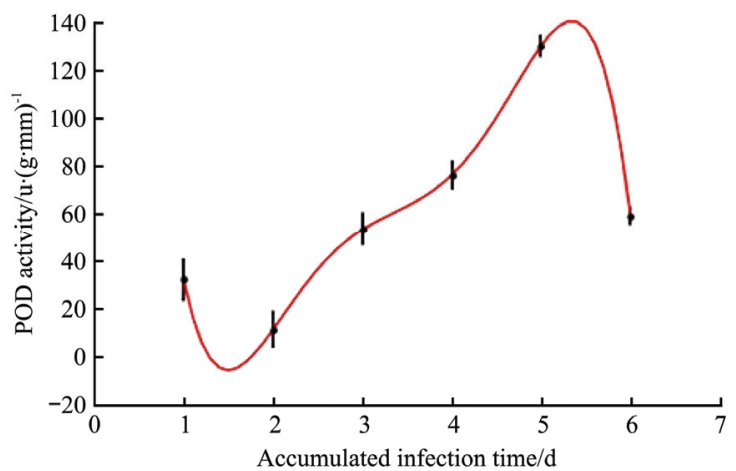

a. Kinetics models of QILONG

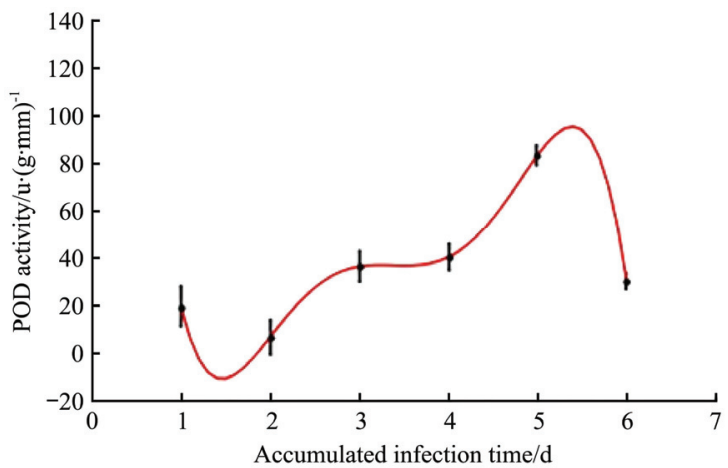

b. Kinetics models of QINGTIAN

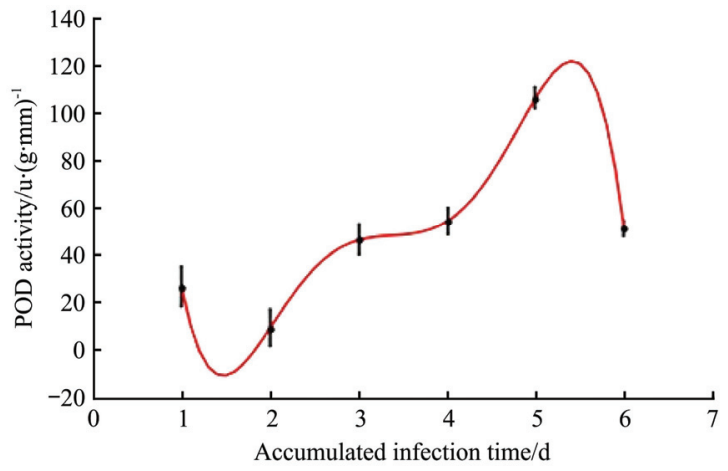

c. Kinetics models of XIAZHAI8

Figure 4 Kinetics models established by the five-order reaction model

Based on the measured POD activities and the initial POD activity values of the infected potato leaves predicted by a hyperspectral model, the kinetic model of POD activity for potato was determined as follow:

Five-order reaction:

$P O D_{1}=-2.8 t^{5}+47.8 t^{4}-308.1 t^{3}+939.8 t^{2}-1312.6 t^{1}+668.0$

$P O D_{2}=-2.8 t^{5}+46.9 t^{4}-300.4 t^{3}+900.6 t^{2}-1228.3 t^{1}+603.0$

$P O D_{3}=-3.2 t^{5}+54.0 t^{4}-348.0 t^{3}+1050.0 t^{2}-1442.8 t^{1}+716.0$

where, $P O D_{1}$ is POD activity of XIAZHAI8, $\mathrm{U} \cdot \mathrm{FW} / \mathrm{g} \cdot \mathrm{h} ; P O D_{2}$ is POD activity of QILONG, $\mathrm{U} \cdot \mathrm{FW} / \mathrm{g} \cdot \mathrm{h} ; \mathrm{POD}_{3}$ is POD activity of QINGTIAN, $\mathrm{U} \cdot \mathrm{FW} / \mathrm{g} \cdot \mathrm{h}$; and $t$ is the accumulated infection time $\mathrm{d}$.

\section{Conclusions}

In this study, the prediction model built by SPA-SVM method obtained the best performance, with a $R p$ (correlation coefficient of prediction) value of 0.923 and a RMSEp (root mean square error of prediction) value of 24.326. Five-order kinetics model according to the prediction model were built, and late blight disease can be predicted according to the five-order kinetics model, which provides a theoretical basis for the prediction of latencies of late blight. By combining the hyperspectral data with the storage time, the kinetic model could be used as a non-destructive method for determining the POD activity of infected potato leaves. In addition, the infection degree could be predicted by applying the hyperspectral kinetic model, which indicates that hyperspectral data are feasible for non-destructive determination of POD activity in potato leaves. In conclusion, both the hyperspectral prediction model and kinetic prediction model can predict POD activity in potato leaves with late blight. Though the changes of POD did not show monotonous increasing or decreasing trends, the POD value varies with the development of the disease, which could be used for the physiological research and auxiliary discrimination of diseases. Because the trend of POD changes is not so regular, other external symptoms such as CAT, SOD and SPAD need to be involved to precisely predict the disease.

\section{Acknowledgement}

This research was supported by the Natural Science Foundation of China (31671965) and the project of Key Laboratory of Agricultural Internet of Things, Ministry of Agriculture, China (2017001). The authors sincerely thank Professor Weixing Shan and Mrs Xueni Chen for their contribution in this study.

\section{[References]}

[1] Gu Y H, Yoo S J, Park C J, Kim Y H, Park S K, Kim J S, et al. BLITE-SVR: New forecasting model for late blight on potato using support-vector regression. Computers \& Electronics in Agriculture, 2016; 130: $169-176$

[2] Information Office of the Ministry of Agriculture, Leading the development of potato staple food with scientific and technological innovation. Available at: http://jiuban.moa.gov.cn/zwllm/zwdt/201501/ t20150106_4323476.htm. (in Chinese)

[3] Journal of the Chinese People's Political Consultative Conference, Strategic start of staple food in China. Available at: http://cppcc.people. com.cn/n/2015/0108/c34948-26348803.html. (in Chinese)

[4] Pallavi S, Bhushan J A, Shanker D R, Mohammad P. Reactive oxygen species, oxidative damage, and antioxidative defense mechanism in plants under stressful conditions. Journal of Botany, 2012; Article ID 217037, 26p. http://dx.doi.org/10.1155/2012/217037

[5] Pal R S, Agrawal P K, Bhatt J C. Molecular approach towards the understanding of defensive systems against oxidative stress in plant: A critical review. International Journal of Pharmaceutical Sciences Review \& Research, 2013; 22(2): 131-138.

[6] Künstler A, Bacsó R, Hafez Y M, Király L. Reactive oxygen species and plant disease resistance. Springer International Publishing, 2015; pp.31-50.

[7] Ik-Hwa H, Woobong C. Phytophthora species, new threats to the plant health in Korea. Plant Pathology Journal, 2014; 30(4): 331-342.

[8] Kamoun S, Furzer O, Jones J D, Judelson H S, Ali G S, Dalio R J, et al. The top 10 oomycete pathogens in molecular plant pathology. Molecular Plant Pathology, 2015; 16(4): 413-434.

[9] Dehury B, Sarma K, Sarmah R, Sahu J, Sahoo S, Sahu M, et al. In silico analyses of superoxide dismutases (SODs) of rice (Oryza sativa L). Journal of Plant Biochemistry \& Biotechnology, 2013; 22(1): 150-156.

[10] Leonowicz G, Trzebuniak K F, Zimakpiekarczyk P, Ślesak I, Mysliwakurdziel B. The activity of superoxide dismutases (SODs) at the early stages of wheat deetiolation. Plos One, 2018; 13(3): e0194678.

[11] Champagne C M, Staenz K, Bannari A, Mcnairn H, Deguise J C. Validation of a hyperspectral curve-fitting model for the estimation of plant water content of agricultural canopies. Remote Sensing of Environment, 2003; 87(2-3): 148-160. 
[12] Sonobe R, Wang Q. Hyperspectral indices for quantifying leaf chlorophyll concentrations performed differently with different leaf types in deciduous forests. Ecological Informatics, 2016: 37: 1-9.

[13] Stroppiana D, Boschetti M, Brivio P A, Bocchi S. Plant nitrogen concentration in paddy rice from field canopy hyperspectral radiometry. Field Crops Research, 2009; 111(1): 119-129.

[14] Likar B, Pernuš F, Katrašnik J, Bürmen M, Špiclin Ž. Geometric calibration of a hyperspectral imaging system. Applied Optics, 2010; 49(15): 2813-2818.

[15] Behmann J, Mahlein A K, Paulus S, Kuhlmann H, Oerke E C, Plümer L. Calibration of hyperspectral close-range pushbroom cameras for plant phenotyping. Isprs Journal of Photogrammetry \& Remote Sensing, 2015; 106: $172-182$.

[16] Chen Y N, Sun DW, Cheng J H, Gao W H. Recent advances for rapid identification of chemical information of muscle foods by hyperspectral imaging analysis. Food Engineering Reviews, 2016; 8(3): 336-350.

[17] Zhang Z L. The experimental guide for plant physiology. Beijing: High Education Press, 2008. (in Chinese)

[18] Menn M L, Tocnaye J L B, Grosso P, Delauney L, Podeur C, Brault P, et al. Advances in measuring ocean salinity with an optical sensor. Measurement Science \& Technology, 2011; 22(11): 115202.

[19] Yu C F, Ding Y L, Hui S W, Yu S B, Wang L Q, Shi L, et al. Analysis of influence on the new type aviation lens shutter to the optical transfer function. Acta Optica Sinica, 2014; 34(11): 1112005

[20] Zhang B, Li J, Fan S, Huang W, Zhao C, Liu C, et al. Hyperspectral imaging combined with multivariate analysis and band math for detection of common defects on peaches (Prunuspersica). Computers \& Electronics in Agriculture, 2015; 114(C): 14-24.

[21] Sun Y, Gu X, Sun K, Hu H, Xu M, Wang Z, Pan L. Hyperspectral reflectance imaging combined with chemometrics and successive projections algorithm for chilling injury classification in peaches. Food Science and Technology, 2016; 75: 557-564. (in Chinese)

[22] Xie C, Feng L, Feng B, Li X, Liu F, He Y. Relevance of hyperspectral image feature to catalase activity in eggplant leaves with grey mold disease. Transactions of the CSAE, 2012; 28(18): 177-184. (in Chinese)

[23] Ghosh S, Chakraborty R, Chatterjee G, Raychaudhuri U. Study on fermentation conditions of palm juice vinegar by response surface methodology and development of a kinetic model. Brazilian Journal of Chemical Engineering, 2012; 29(3): 461-472.

[24] Mounira K A, Serge H, Nawel O, Radia C, Noreddine. Kinetic models and parameters estimation study of biomass and ethanol production from inulin by Pichiacaribbica (KC977491). African Journal of Biotechnology, 2017; 16(3): 124-131.

[25] Vašát R, Kodešová R, Klement A, Borůvka L. Simple but efficient signal pre-processing in soil organic carbon spectroscopic estimation. Geoderma, 2017; 298: 46-53.

[26] Huang S, Yan W, Liu M, Hu J. Detection of difenoconazole pesticides in pakchoi by surface-enhanced Raman scattering spectroscopy coupled with gold nanoparticles. Analytical Methods, 2016; 8(23): 4755-4761.

[27] Koseki J, Kita Y M. Formation of Schiff-base for photoreaction mechanism of red shift of GFP spectra. Biophysical Chemistry, 2010; 147(3): 140-145. 